\title{
Study of the Ardon deep fault spatial position using high-precision gravity and magnetic measurements
}

\author{
Vladislav Zaalishvili1', Aleksandr Kanukov, Zarina Persaeva, and Georgi \\ Shmanatov
}

Geophysical Institute - the Affiliate of Vladikavkaz Scientific Center of the Russian Academy of Sciences, 362002, Vladikavkaz, Russia

\begin{abstract}
Gravity prospecting (gravimetry) is one of the geophysical methods for studying the upper part of the Earth crust. To study the spatial position of the Ardon deep fault, the studies on the gravitational field gradient change have been carried out. A site along the road of Arkhonskaya - Dur-Dur was chosen as a profile. According to the obtained results, the greatest deviation from the average value is observed on the section from the 18 th to the 36 th kilometer. At the same time, there are areas with the values close to the average. This may indicate the presence of several "branches" of the fault. A similar profile was traversed using a magnetometer in order to compare the results with the data obtained by the gravimeter. According to the geomagnetic field modulus values, two areas are also singled out. Probably these areas are the extensions of the Ardon deep fault "branches", found in early studies on the Upper ZgidprofileDzhimidon field with the help of the micro-seismic sounding method (MSM). According to the obtained data, it is possible to conclude that the gravimetric measurements, as well as the magnetic ones, allow determining the places of the probable spatial location of the faults. For a more detailed study of the Ardon deep fault, its further study with the help of MSM is proposed.
\end{abstract}

\section{Introduction}

Gravity prospecting (gravimetry) is one of the geophysical methods for studying the upper part of the Earth crust; the purpose of which is prospecting and exploration of mineral deposits [1-3]. Gravity prospecting is an applied part of a wider discipline from the field of geophysics - gravimetry, which deals with the measurement of gravity to solve scientific and applied problems in determining the shape of the Earth, studying the change in the potential of gravity over time, solving various problems of geodynamics [4-11], monitoring seismically active zones [12-14] etc.

\footnotetext{
${ }^{1}$ Corresponding author: cgi_ras@mail.ru
} 
Rocks' differentiation by density underlies the physical basis of gravity prospecting. In the case when the rocks, that compose the Earth crust, are heavier in relation to those rocks, that surround a certain area, the force of gravity will increase in this zone, since a positive anomaly will be observed here; otherwise, a negative anomaly will form. At the same time, gravity anomalies of both types also occur in the zones of possible earthquake sources (PES). Determination of the PES zones parameters [14-15] is the inverse problem of gravity prospecting, which consists in determining the parameters of anomalous bodies by the measured distribution of the gravitational field. The inverse problem (in contrast to the direct problem) has not the only solution, as a specified distribution of the gravitational field can correspond to countless variants of the distribution of both excess and insufficient masses. The uncertainty degree is reduced if the researcher uses the data from other geophysical methods.

A number of studies [16-20] reveal the necessity of accounting the anomalous gradient, which is caused by the difference in heights of observation points in relation to the anomalous masses occurring primarily in the upper part of the section. Model examples show that the value of the anomalous gradient can range from $0.05 \mathrm{mGal} / \mathrm{m}$ to $0.5 \mathrm{mGal} / \mathrm{m}$ $[13,17]$.

A special pedestal was developed to conduct the field studies on measuring the gravitational field gradient (Figure 1).

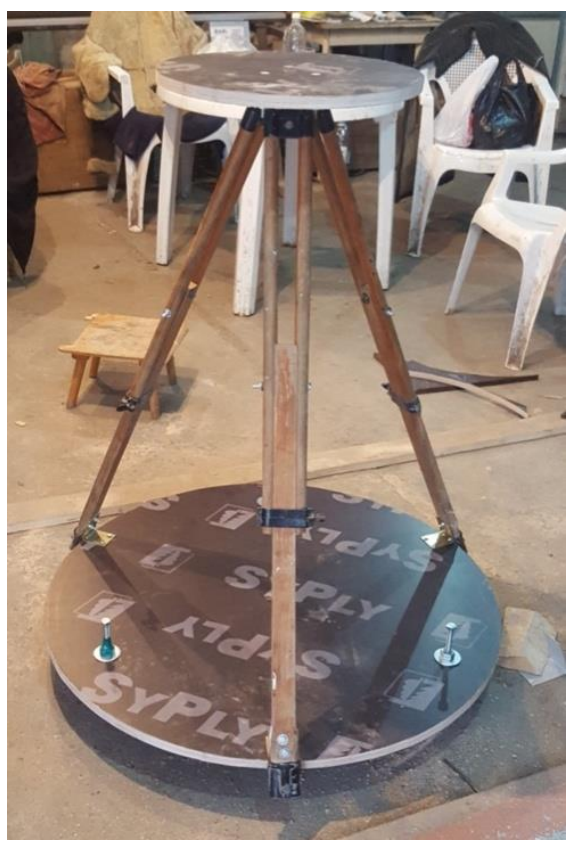

Fig. 1. The process of the pedestal assemblage

The pedestal consists of two plane-parallel platforms for the gravimeter setting up, the distance between which is equal to 1 meter. Gravity gradient measurements can be made using only a tripod, but in this case, the height of the site will differ from one point of measurement to another. This creates additional inconveniences since it becomes necessary to measure accurately the height of the gravimeter installation in the upper position relative to the lower one. The great advantage of the special pedestal is that there is no need for a time-consuming installation of the device because the height difference is constant and is estimated equal to one meter. This positively effects the results of the field data processing, 
since the value of the gradient can be calculated by simply subtracting the gravity values in different positions, and the resulting value will already be the gravity gradient value per meter. Moreover, the pedestal has three adjusting screws that allow setting it strictly on the level.

\section{Methods}

For studying the spatial location of the Ardon deep fault, a profile was selected along the road of Arkhonskaya - Dur-Dur, with a step of 500 meters. Thus, 82 observation points were identified (Figure 2).

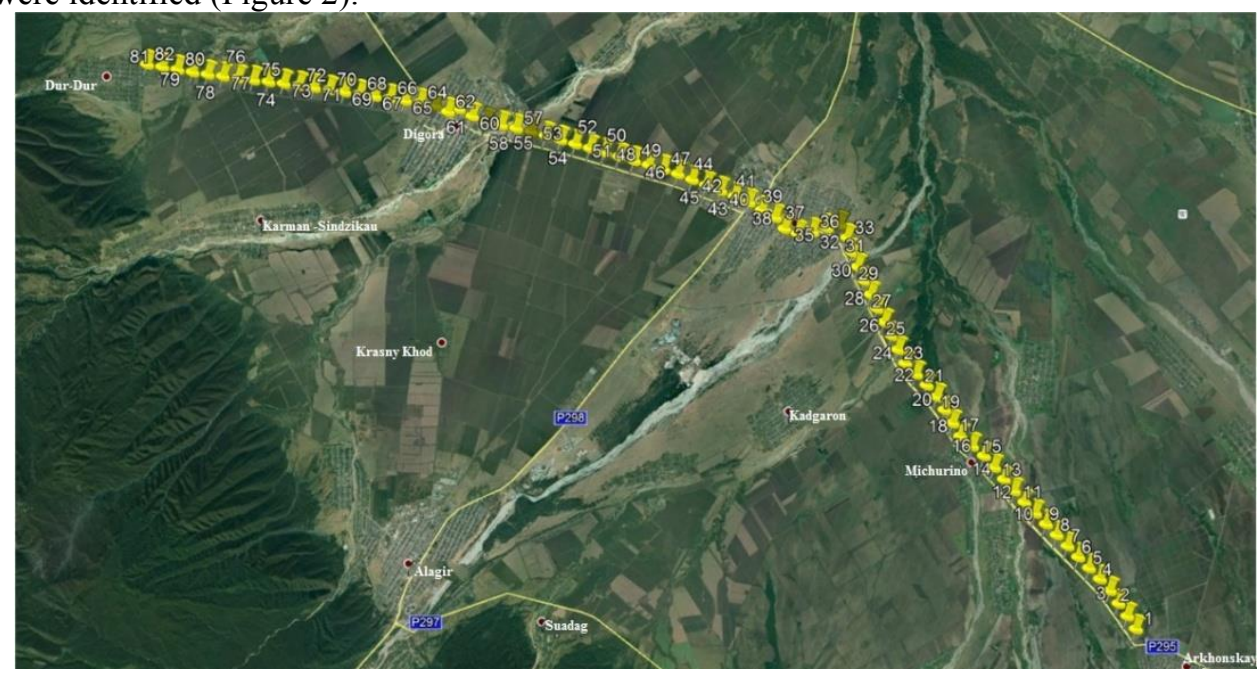

Fig. 2. Profile for the Ardon deep fault spatial position study

The measurements were also realized according to the scheme of the repeated bonds of the A-B-A type. Herewith, 5 measurements were recorded at a lower point and 4 measurements at the upper point. Thus, 7 gradient subsequently averaged values were calculated at one point.

Besides, the corrections, taking the working and stationary drift of the equipment [2122] during measurements into account, were calculated according to the following methodology. Let us denote the gravity force at the two studied points as $g_{1}^{t_{1}}, g_{2}^{t_{1}}$, and the increment of the force of gravity between the two points measured at the $\mathrm{t}_{1}$ period as $\Delta p^{t_{1}}=g_{1}^{t_{1}}-g_{2}^{t_{1}}$. Similarly $g_{1}^{t_{1}}, g_{2}^{t_{1}}$, and $\Delta p^{t_{1}}=g_{1}^{t_{1}}-g_{2}^{t_{1}}$ are the same quantities that were measured at the $t_{2}$ period. According to the introduced designations, the increment of the gravity force between the considered points over the time interval $\Delta t=t_{2}-t_{1}$, is expressed by the following formula:

$$
\begin{gathered}
\Delta p=\Delta p^{t_{2}}-\Delta p^{t_{1}}=\left(g_{1}^{t_{2}}-g_{2}^{t_{2}}\right)-\left(g_{1}^{t_{1}}-g_{2}^{t_{1}}\right)=\left(g_{1}^{t_{2}}-g_{2}^{t_{1}}\right)-\left(g_{2}^{t_{2}}-g_{2}^{t_{1}}\right)= \\
=\Delta g_{1}-\Delta g_{2}=\left(\Delta \widetilde{g_{1}}+\Delta h_{1} * \widetilde{W_{1}}\right)-\left(\Delta \widetilde{g}_{2}+\Delta h_{2} * \widetilde{W_{2}}\right)
\end{gathered}
$$

where:

$\Delta g_{i}=\left(\Delta \widetilde{g_{l}}+\Delta h_{i} * \widetilde{W_{1}}\right)$ is the summand related to the change in the gravitational field at the point $\mathrm{i}(\mathrm{i}=1,2)$ during the time $\Delta \mathrm{t}$ due to the difference in heights between two points $\Delta \mathrm{h}_{\mathrm{i}}$ and the difference in the values of the gravitational field $\Delta \widetilde{\mathrm{g}}_{1}$, caused by the difference in the geological structure at the points under study 
$\widetilde{W_{l}}=W_{i}+2 \pi G \sigma$

Where: $W_{i}$ is the vertical gradient of gravity measured at point $i$; $2 \pi G \sigma$ is Bouguer correction $\left(2 \pi G \sigma \approx 113 \mathrm{mcGal} / \mathrm{m}\right.$ at a density $\left.\sigma \approx 2.7 \mathrm{~g} / \mathrm{cm}^{3}\right)$; $G$ is a gravitational constant.

In the case when $\Delta \widetilde{g}_{\imath} \approx 0$, i.e. the gravitational field changes insignificantly between two points, without taking the change in the field due to the change in height into account, the increment of the force of gravity between the points is expressed by the following formula:

$$
\Delta p=\Delta h_{1} * \widetilde{W_{1}}-\Delta h_{2} * \widetilde{W_{2}}
$$

\section{Results}

The measurements were carried out at all points of the selected profile (Figure 3). The graph of the gravitational field gradient behavior along the profile of Arkhonskaya - DurDur is shown in Figure 4. It is also necessary to note the high accuracy of the performed measurements: the deviation of the values during measurements, as a rule, did not exceed one mcGal.

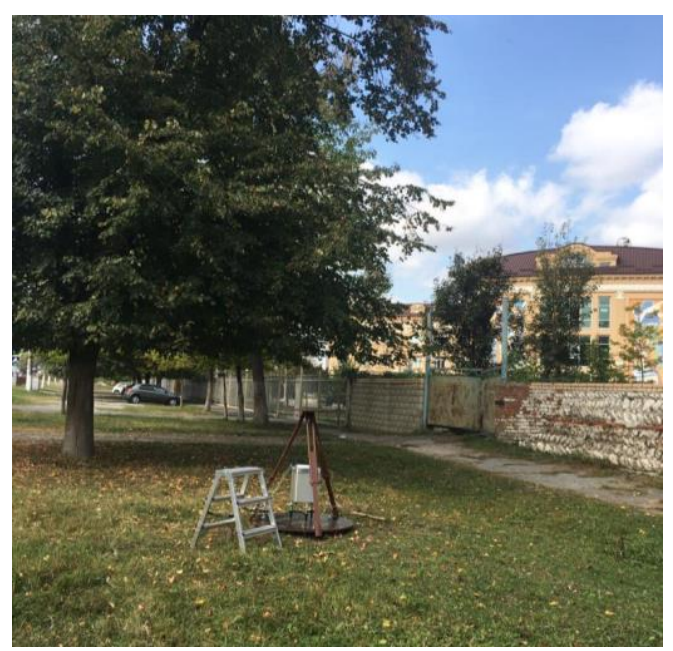

Fig. 3. Field measurements of the gravitational field gradient

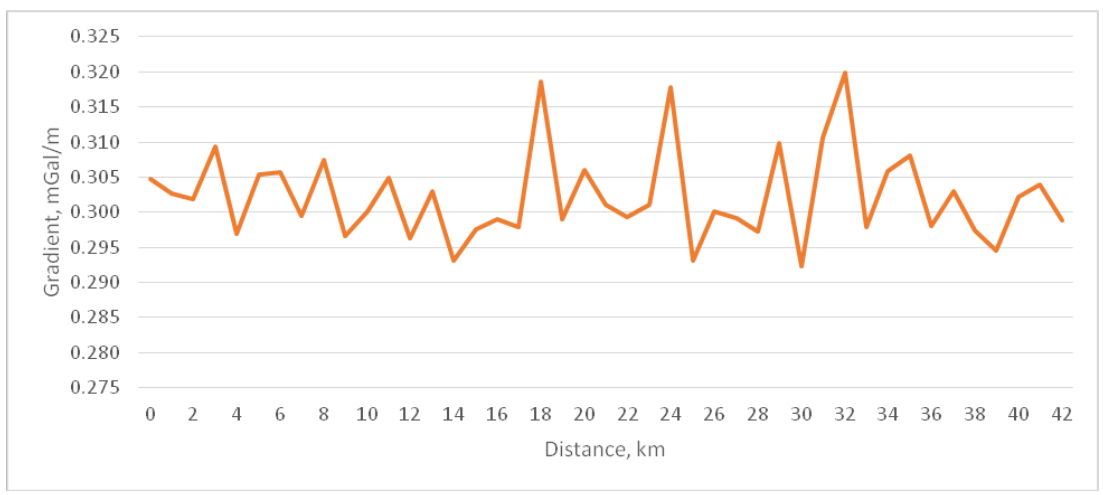

Fig.4. Graph of the behavior of the gravitational field gradient along the profile of Arkhonskaya Dur-Dur 
Using the data of the presented graph, it is possible to see that the greatest deviation from the average value is observed for the section from the 18th to the 36th kilometer. At the same time, there are areas where the values are close to the average ones, which may indicate the presence of several "branches" of the fault.

Moreover, as was noted above, the degree of uncertainty in the results of gravimetric measurements is reduced if the researcher uses the data from other geophysical methods. In this regard, an additional study of this profile was carried out using magnetic measurements.

The profile under consideration was traversed using a magnetometer in order to compare the results with the data obtained by the gravimeter. Figure 5 shows the measurement results.

As it is shown in Figure 5, two areas are also singled out in this profile. Probably they are the extensions of the "branches" of the Ardon deep fault, found in early studies on the profile of the Upper Zgid - Dzhimidon field with the help of the micro seismic sounding method (MSM) [17]. Figure 6 shows a combined graph of the behavior of the gravitational field gradient and the module of the geomagnetic field.

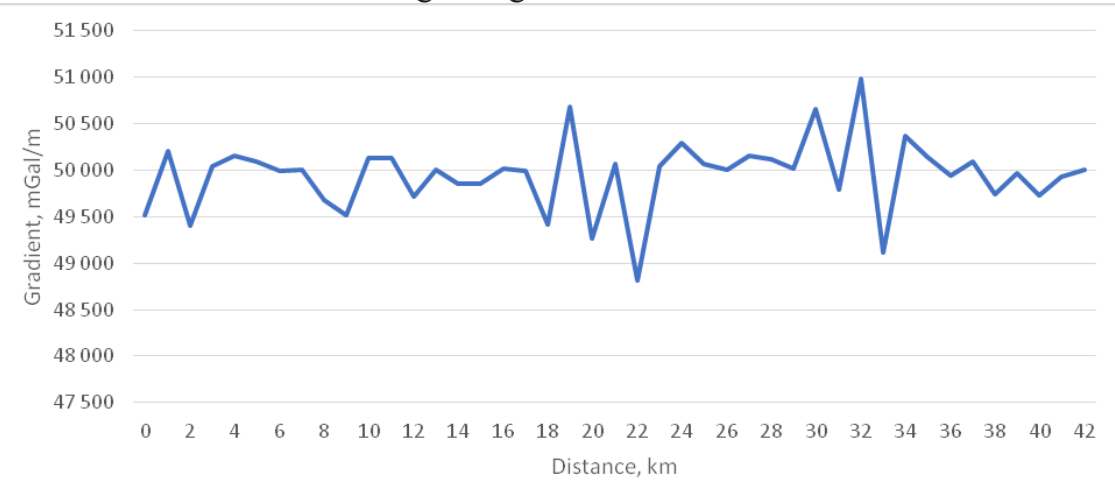

Fig. 5. Measurement results of the geomagnetic field module using the MMPOS-1 magnetometer on the profile of Arkhonskaya - Dur-Dur

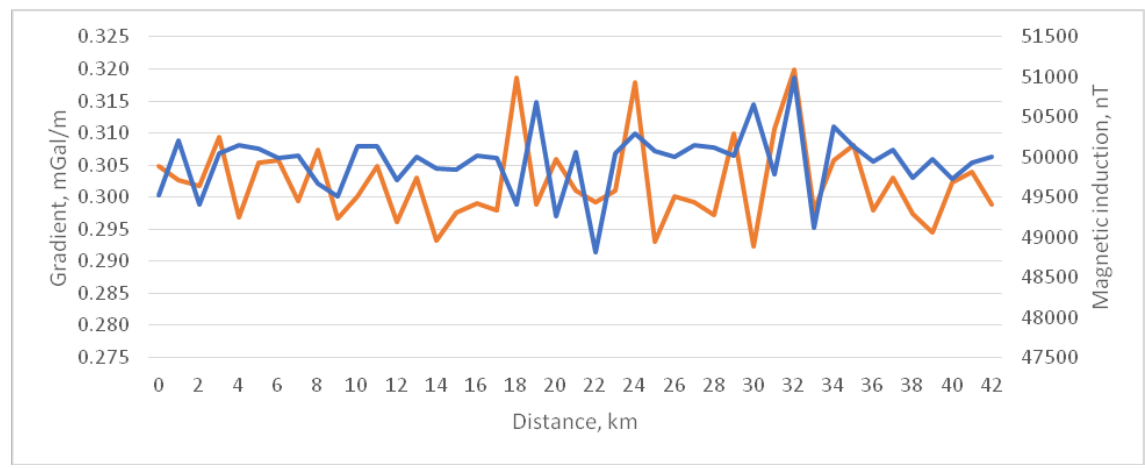

Fig. 6. Combined graph of the behavior of the gravitational field gradient and the modulus of the geomagnetic field on the profile of Arkhonskaya - Dur-Dur (blue color denotes magnetometer)

Using the obtained data, it is possible to conclude that gravimetric measurements, as well as magnetic ones, allow determining the places of the probable spatial location of the faults. For a more detailed study of the Ardon deep fault, its further study with the help of MSM is proposed. 


\section{Summary}

- To study the spatial location of the Ardon deep fault, a profile was selected along the road of Arkhonskaya - Dur-Dur, with a step of 500 meters. Thus, 82 observation points were identified. The measurements were carried out according to the scheme of the repeated bonds of the A-B-A type.

- The greatest deviation from the average value for the gravitational field gradient is observed on the section from the 18th to the 36th kilometer. At the same time, there are areas where the values are close to the average. This may indicate the presence of several "branches" of the fault.

- Using a magnetometer, the profile of Arkhonskaya - Dur-Dur was traversed in order to compare the results with the data obtained by the gravimeter. Two areas are also singled out in this profile. They probably are the extensions of the "branches" of the Ardon deep fault, found in early studies on the profile of the Upper Zgid - Dzhimidon field with the help of the micro seismic sounding method.

- Using the obtained data, it follows that gravimetric measurements, as well as magnetic ones, allow determining the places of the probable spatial location of the faults. For a more detailed study of the Ardon deep fault, its further study with the help of MSM is proposed.

\section{References}

1. K. Khulelidze, Yu. Kondratiev, V. Zaalishvili, Z. Betrozov, Sustainable Development of Mountain Territories 8 (1), 46-51 (2016).

2. G. Grigorkina, A. Ramonova, D. Kibizov, E. Kozyrev, V. Zaalishvili, T. Magkoev, K. Fukutani, Solid State Communications 257, 16-19 (2017).

3. V.K. Milyukov, V.D. Iushkin, A.P. Mironov, V.B. Zaalishvili, A.S. Kanukov, B.V. Dzeranov, Geology and Geophysics of Russian South 3 (2), 39 - 45 (2020). DOI: 10.23671/VNC.2013.2.55543

4. A. Gorbatikov, E. Rogozhin, M. Stepanova, Yu. Kharazova, N. Andreeva, F. Perederin, V. Zaalishvili, D. Melkov, B. Dzeranov, B. Dzeboev, A. Gabaraev, Physics Earth 1, 2839 (2015).

5. V. Zaalishvili, D. Melkov Izvestiya, Physics of the Solid Earth 50 (5), 707-718 (2014).

6. V. Zaalishvili, A. Kanukov, D. Melkov, V. Makiev, L. Dzobelova, International Journal of GEOMATE 15 (51), 160-166 (2018).

7. I.A. Kerimov, Geology and Geophysics of Russian South 10 (10), 55-67 (2020). DOI: 10.23671/VNC.2020.1.59065

8. T.V. Giorgobiani, Geology and Geophysics of Russian South 10 (1), 35-42 (2020). DOI: 10.23671/VNC.2020.1.59063.

9. G.S. Yanvarev, Geology and Geophysics of Russian South 10 (4), 31 - 40 (2020). DOI: 10.46698/VNC.2020.55.16.002

10. Kh.O. Chotchaev, O.G. Burdzieva, V.B. Zaalishvili, Geology and Geophysics of Russian South 10 (4), 70 - 100 (2020). DOI: 10.46698/VNC.2020.87.26.005.

11. A.A. Drobinskiy, N.N. Golchikova, P.P. Kamnev, Geology and Geophysics of Russian South 10 (3), 114 - 125 (2020). DOI: 10.46698/VNC.2020.13.71.007

12. V. Zaalishvili, D. Melkov, B. Dzeranov, F. Morozov, G. Tuaev, International Journal of GEOMATE 15 (47), 158-163 (2018). 
13. V. Zaalishvili, N. Nevskaya, D. Melkov, Izvestiya. Physics of the Solid Earth 50 (2), 263-272 (2014).

14. V. Zaalishvili, E. Rogozhin, Open Construction and Building Technology Journal 5, 30-40 (2011).

15. V. Zaalishvili, D. Melkov, A. Kanukov, B. Dzeranov, International Journal of GEOMATE 10 (1), 1656-1661 (2016).

16. L. Nemtsov, Nedra (1967).

17. A. Lyubimov, Nedra (1979).

18. Nikolsky, Rudgeofizika (1981).

19. S. Serkerov, A. Koptunov, A. Lyanders, Oil and Gas Studies 6, 24-30 (2006).

20. A. Lyanders, Oil and Gas Studies 5, 20-23 (2007).

21. V. Milyukov, V. Yushkin, A. Mironov, V. Zaalishvili, A. Kanukov, B. Dzeranov, Geology and Geophysics of Russian South 2, 39-45 (2013).

22. V. Milyukov, V. Yushkin, A. Kopaev, A. Mironov, G. Dem'Yanov, R. Sermyagin, A. Basmanov, V. Popad'Ev, I. Nasretdinov, V. Zaalishvili, A. Kanukov, B. Dzeranov, Measurement Techniques 56 (10), 1105-1110 (2014).

23. V. Zaalishvili, Kh. Chotchaev, Geology and Geophysics of Russian South 4, 52-67 (2016). 